\title{
PROCEDIMIENTOS PARA EL DESARROLLO RURAL DE LA REGIÓN DE MURCIA (HORIZONTE: 2006)
}

\author{
Mercedes Millán Escriche \\ Escuela Universitaria de Turismo de Murcia
}

\section{RESUMEN}

En este artículo se analizan los procedimientos que constituyen el eje primordial para alcanzar el desarrollo en los ámbitos rurales de la Región de Murcia. Con este fin se examinan las fuentes que proporcionan métodos, definiciones y conceptos afines con el tema de estudio, a la par que ofrecen una visión amplia de la problemática rural y de sus particularidades. Asimismo, se revisan normas, decisiones, programas y recomendaciones de los organismos y administraciones implicados en el desarrollo rural.

Palabras clave: procedimientos, desarrollo rural, fuentes, normas, programas,...

\section{ABSTRACT}

This article analyses the procedures that constitute the primordial axis to reach the development in the rural environments of the Region of Murcia. With this aim the sources that provide methods, definitions and similar concepts with the subject of the study are examined, at par that they offer a wide view of the rural problem and of their particularities. Likewise, norms, decisions, programs and recommendations of the organisms and administrations implied in the rural development are revised.

Key words: procedures, rural development, sources, norms, programs,...

\section{Introducción}

En la Región de Murcia, un espacio que desde hace décadas es objetivo de las acciones que propician el desarrollo es el Noroeste. Este territorio, en cuyo estudio ya hemos ahondado en trabajos anteriores citados en la bibliografía, como otros ámbitos rurales, no goza de idéntico nivel de progreso que otros territorios de la misma Comunidad Autónoma y, por esa razón, deben tomarse en consideración las propuestas derivadas de las políticas comunitarias, cuyo fin es modificar la actual trayectoria negativa de estas poblaciones, 
impulsar la participación de sus habitantes en las iniciativas de desarrollo y lograr que las regiones coexistan en la igualdad.

Los procedimientos de dichas políticas se vienen aplicando, con especial énfasis, a partir de la puesta en funcionamiento de la Iniciativa Comunitaria de Desarrollo Rural LEADER, que no debe desmembrarse de un tronco mucho más complejo: la Política Agraria Comunitaria y los Fondos que atienden a su financiación. En este contexto, la última década puede considerarse rupturista, debido a que se intentan llevar a determinados espacios rurales proyectos innovadores que no siempre están estrechamente relacionados con la actividad más tradicional del medio: la agricultura. Se pretende que sea la propia población rural la que tome la iniciativa, que sea la que decida lo que quiere para su espacio social, económico y, en definitiva, vital, pero el camino a recorrer es particularmente difícil porque los cambios suelen generar desconfianza y sólo las referencias de buenos resultados obtenidos en otros espacios rurales, con problemáticas similares, pueden significar el revulsivo necesario para dinamizar a la población local.

Las reflexiones anteriores constituyen la hipótesis de este trabajo y nuestro objetivo es obtener una serie de respuestas al analizar los procedimientos que constituyen el eje primordial para alcanzar el desarrollo en los ámbitos rurales de la Región de Murcia. Esta intención requiere buscar previamente fuentes de autores e instituciones que proporcionan métodos, definiciones y conceptos afines con el tema de estudio, a la par que ofrecen una visión amplia de la problemática rural y de sus particularidades. Asimismo, se trata de revisar normas, decisiones, programas y recomendaciones de los organismos y administraciones implicados en el desarrollo rural. Sin embargo, una lógica adecuación del contenido a la extensión de este trabajo, impide retroceder excesivamente en el tiempo para buscar indicios de acciones que han pretendido mejorar la situación del medio rural murciano. Esta es la razón por la que se estudia sólo el periodo transcurrido desde principios de los noventa hasta 2006, un periodo, no obstante, que coincide con el momento en el que con mayor énfasis se ha proclamado que el desarrollo rural constituye un pilar fundamental en la P. A. C. Buena prueba de ello es la creación de una Iniciativa Comunitaria, LEADER, especialmente dedicada a favorecerlo.

\section{Acciones a favor del desarrollo rural en la Región de Murcia. Antecedentes}

Resulta conocido que la Región de Murcia ha estado adscrita al Objetivo $n^{\circ} 1$ por su situación desfavorable, en términos de renta, respecto a otros espacios de la Unión Europea. Esta circunstancia tiene su reflejo en los sucesivos Programas Operativos (en adelante P. O.), en los que se han incorporado nuevas estrategias a los ejes de desarrollo que los integran. Así, la Decisión de la Comisión C (90) 2583/90 de 14 de diciembre de 1990 aprobó el P. O. de la Región de Murcia para el periodo 1990-1993 y concentró sus actuaciones en la dotación de infraestructuras básicas, tratando con ello de propiciar la ruptura del tradicional aislamiento de la Región, respecto a los principales focos económicos, e intentando vertebrar el territorio interior para favorecer el desarrollo del potencial endógeno y facilitar el acceso a las distintas áreas. Por otra parte, se intentó adecuar, modernizar y diversificar el tejido productivo regional mediante la Subvención Global junto a los recursos del FEDER.

Conviene destacar que el periodo de vigencia, establecido en un principio para 19901993, quedó definitivamente marcado desde agosto de 1990 hasta el 30 de junio de 1994, con un total para la Subvención Global de 5.022,25 millones de pesetas, de los que 2.775,36 correspondieron al FEDER. 
De 1994 a 1999 el Marco Comunitario de Apoyo contempló 12 P. O. regionales correspondientes a otras tantas Comunidades Autónomas Objetivo 1 que comparten una estrategia global pero que tienen especificidades y estrategias particulares. Entre sus ejes, el dedicado a «agricultura y desarrollo rural» recoge aspectos como la prestación de servicios a las explotaciones agrarias, comercialización de productos agrarios de calidad e ingeniería financiera. Esta medida se propone al amparo del artículo 33, $4^{\circ}$ guión, del Reglamento (CE) 1257/1999 del Consejo, de 17 de mayo de 1999, sobre la ayuda al desarrollo rural a cargo del FEOGA por el que se modifica y derogan determinados reglamentos.

Hay que señalar, en esta etapa, la concesión de ayudas destinadas a mejorar la competitividad y, en general, desarrollar el tejido productivo del sector agroalimentario en la Región de Murcia. Con la aportación del FEOGA-O de 8.622,34 millones de pesetas, la de la Administración Central de 1.668,1 millones y la de la Administración Autonómica de 2.025,07, elevan la cifra destinada a este Programa a 12.315,2 millones de pesetas. La forma de actuación es a través de tres subprogramas:

1. Mejora de las condiciones agrarias (con particular énfasis en la mejora de regadíos);

2. Protección y conservación de los recursos naturales;

3. Reconversión, reorientación y mejora de producciones agrarias. Se pretende optimizar el uso del agua y de los fertilizantes, reestructurar el sector hortofrutícola, mejorar la sanidad vegetal y animal y fomentar la comercialización y transformación de productos agrarios en régimen asociativo.

En el P. O. existe, asimismo, un especial interés por la concesión de ayudas para potenciar la creación y apoyar el funcionamiento, control e inversiones de los Consejos de las Denominaciones de Origen, Genéricas y Específicas; Agricultura Ecológica; marcas de Calidad y organismos encargados de la Certificación de las características específicas de los productos agroalimentarios; así como las Organizaciones Interprofesionales Agroalimentarias; las Agrupaciones de empresas; e incluso las empresas dedicadas a la comercialización y transformación de productos agroalimentarios que inicien actuaciones destinadas a lograr el aseguramiento de la calidad, conforme a normas oficiales, y a desarrollar denominaciones y marcas de calidad.

El Informe de Evaluación del P. O. FEDER de la Región de Murcia, 1994-1999, estima que existe un alto nivel de coherencia de las actuaciones y proyectos incluidos en el mismo, con respecto a las prioridades y necesidades contempladas en el Plan de Desarrollo Regional (P. D. R.) de Murcia (1994-1999) y con las orientaciones del Marco de Apoyo Comunitario (M. A. C.) 1994-1999. Se consideran convenientes las acciones llevadas a cabo durante el primer trienio de vigencia, ya que éstas inciden en las posibilidades de desarrollo de la Región a través de la mejora de las infraestructuras y de los equipamientos básicos, además de la ampliación de los equipamientos sanitarios y educativos. No obstante, en el Informe se hace hincapié en que las necesidades en esas materias son todavía amplias en la Región, siendo necesaria la continuidad de los Fondos Estructurales para seguir corrigiendo las insuficiencias existentes. Por otra parte se subraya la incidencia positiva de dichos Fondos y su contribución a la corrección de los estrangulamientos y desequilibrios existentes, aunque su repercusión en la economía regional no sea cuantitativamente elevada.

Es recomendable una mayor concentración de la inversión elegible en un menor número de proyectos de alto contenido estratégico, en lugar de la diversificación practicada, a fin 
de cuantificar de forma más clara el impacto del Programa. No obstante, se reconoce que la dinámica seguida ha permitido contribuir, en mayor medida, a la corrección de algunos desequilibrios que sufre la Región. En síntesis, del análisis de coherencia se deduce una continuidad, complementariedad e intensificación de las acciones emprendidas en la anterior etapa, en sintonía con las propuestas de los agentes económico-sociales de Murcia y con los diferentes planes existentes en la Región.

Hay que advertir, que si bien en el plano económico no se observan cambios relevantes, con respecto a los desequilibrios territoriales existentes, se aprecia una mejora creciente del sector rural en general. La disponibilidad de recursos financieros para el ámbito rural, ejerce un efecto positivo y permite la aparición de nuevas formas de intervención en el mundo agrario. Otro de los efectos detectados es la mejora del aprovechamiento de los recursos hídricos, reduciéndose las pérdidas de agua para riego gracias a la contribución que ha supuesto el gasto con cargo al Programa Operativo, lo que supone una mejora en el rendimiento del agua disponible y una disminución de los costes de producción.

En los trabajos de seguimiento y evaluación final del Programa Operativo 1994-1999, se observa que la Renta Agraria desde el año 1993 se incrementó en un 55\%. Este aumento se repartió a lo largo de todos esos años, siendo más importantes los incrementos en 1996 y 1998. Sin embargo, la evolución de la Renta Agraria generada por persona ocupada en el sector ha presentado un comportamiento diferente, mostrando un crecimiento desde el año 1993 del 25,5\%, pero este incremento no ha sido constante en todos estos años, mostrando un fuerte aumento en el año 1996, y unos descensos en años posteriores que se imputan a las siguientes causas:

- Los buenos resultados de la Renta Agraria generada por persona en el año 1996 debido a la baja ocupación del empleo agrario.

- Los no tan altos resultados de la Renta Agraria por persona en los años 1998 y 1997, debido al fuerte aumento de la ocupación del empleo agrario.

Respecto a la Producción Final Agraria (P. F. A.), la variación desde 1989 ha experimentado una evolución desigual para cada uno de los subsectores. El subsector agrícola suponía en torno al 70\% de la P. F. A. Desde el año 1990 hasta el 1992 había experimentado un continuo descenso, hasta que, a partir del año 1993, ha ido en continuo ascenso, mostrando rangos de crecimiento diferentes cada año debido a las variaciones que han existido en los factores de producción. En los años 1995, 1996 y 1997 se produjo un contenido aumento, y entre 1997 y 1998 el incremento fue del 6,9\%.

Junto a las acciones que han derivado en los resultados anteriores, la década de los noventa se identifica con la Iniciativa Comunitaria para el Desarrollo Rural LEADER. Dicha Iniciativa tuvo dos fases que, en la Región de Murcia, presentaron notables diferencias, tanto en el espacio regional afectado por sus beneficios, como por la naturaleza de las actuaciones. De forma muy sintetizada, recordar que LEADER I se implanta exclusivamente en el Noroeste murciano, con un único Grupo de Acción Local (GAL), mientras que LEADER II actuó en un territorio mucho más amplio que fue gestionado por tres GAL.

LEADER I se desarrolló entre 1992 y 1994 e hizo posible inversiones de más de 1.140 millones de pesetas recibidas de la Comunidad Europea, la Administración Central, la Autonómica y las locales, así como la procedente de la iniciativa privada. Este capital se destinó preferentemente a actuaciones como: apoyo técnico de iniciativas locales; actividades de formación y empleo; valorización y comercialización de los productos locales, 
desarrollo del turismo y creación de nuevas empresas. Las actuaciones más relevantes son las relacionadas con parajes naturales y educación ambiental. Las actividades deportivas en el medio rural, con vistas a un Turismo Activo/Deportivo, se potenciaron mediante la creación de un centro hípico en Caravaca y cuadras con caballos en Las Oicas, Archivel. Para la promoción turística comarcal fue relevante la creación de una Central de Reservas Comarcal; la edición de una guía turística comarcal con base histórica y patrimonial; la edición de una guía turística del Noroeste y actuaciones de promoción y divulgación del turismo rural.

La necesidad de formar para adaptar a las nuevas actividades en el medio rural se plasmó en las «Jornadas Europeas sobre rehabilitación de edificios tradicionales con destino turístico»; «Jornadas Europeas sobre historia y patrimonio como recursos turísticos en zonas rurales» y en el curso de gestión de alojamientos rurales.

Una medida más, susceptible de ayudas LEADER, es la asistencia técnica y, en este sentido, LEADER I financió los Estudios y Proyectos del Parque Ecológico Recreativo de Cehegín; un Estudio espeleológico de adecuación turística de la Cueva del Puerto en Calasparra; la creación del equipo LEADER de arquitectura para asistencia en rehabilitaciones y el Inventario de edificios rurales con potencial turístico.

Además de esta línea de acciones eminentemente orientadas hacia el turismo, se realizaron otras que potencian a la artesanía y las PYMES. La valorización de las producciones agrarias se llevó a cabo a través de intervenciones como la instalación de una quesería de cabra en Campos del Río; un obrador de repostería artesanal en Caravaca; el incremento de colmenas para apicultura en Caravaca; la regulación de riegos y construcción de embalse para cultivos biológicos a gran altitud en Inazares (Moratalla) y la ampliación de una bodega en Tercia de Ulea (Moratalla), entre otras actuaciones.

LEADER II fue mucho más prolijo en el número de proyectos y, por esa razón, obviamos su detalle en este trabajo. Simplemente señalar que, entre los tres GAL existentes en esta fase, se ejecutaron en torno a los 4.900 millones de pesetas de inversión que se materializaron en casi 450 proyectos de naturaleza variada ${ }^{1}$.

\section{Procedimientos para el desarrollo rural en la Región de Murcia (2000-2006)}

La continuidad a los Programas Operativos anteriores se encuentra en la Decisión de la Comisión, C(2000)2552 de 19 de octubre de 2000, por la que aprueba el Marco Comunitario de Apoyo para las Regiones Objetivo 1, es decir, se renueva el instrumento financiero de ayudas para ellas. En esta ocasión el Marco Comunitario de Apoyo español está compuesto por 23 Programas Operativos, de los cuales 12 son regionales y 11 plurirregionales. Su aplicación es de 2000 a 2006.

El Programa Operativo Regional, de este periodo, se articula en 9 ejes prioritarios que son convenientemente adaptados a las especificidades y estrategias regionales que articulan la estrategia global del conjunto de las regiones pertenecientes al Objetivo $\mathrm{n}^{\circ}$ 1. Para esta aportación destacamos por su estrecha relación el eje 7 «Agricultura y Desarrollo Rural», cuyas actuaciones se articulan a través de las siguientes medidas (cuadro $\mathrm{n}^{\circ} 1$ ):

1 Se puede ver la relación completa de los proyectos ejecutados en: Desarrollo rural en la Región de Murcia, Leader II 1994-1999. CONSEJERÍA DE AGRICULTURA, AGUA Y MEDIO AMBIENTE, Dirección General de Regadíos y Desarrollo Rural. 
$>$ Desarrollo y mejora de las infraestructuras de apoyo:

Adecuación y mejora de la red de caminos rurales de la Región, con el fin de facilitar las comunicaciones y el acceso a las explotaciones agrarias para el aporte de materias primas, productos, etc. mejorando la eficacia de las mismas; y la dotación de accesos a caseríos, cortijos y viviendas rurales diseminadas, lo que mejora la calidad de vida de los agricultores y evita el despoblamiento de determinadas zonas.

$>$ Prestación de servicios a las explotaciones agrarias, comercialización de productos agrarios de calidad e ingeniería financiera

Con esta medida se pretende potenciar la creación, el funcionamiento y el apoyo a las inversiones realizadas por las Denominaciones de Origen, específicas y genéricas; las Organizaciones Interprofesionales Agroalimentarias; los Consejos de Agricultura Ecológica; los que amparen Marcas de Calidad y las Asociaciones y Agrupaciones de Empresas e incluso las empresas a título individual, que estén acogidas a cualquiera de los Consejos, Organizaciones o la Marca de Calidad «Calidad Agroalimentaria». Es decir, muestra una clara continuidad con la estrategia del P. O. anterior.

Por lo que se refiere al avance de la ejecución física, cuyos resultados se observan en el Cuadro $\mathrm{n}^{\circ} 1$ con los indicadores relativos al desarrollo rural, en términos generales, la mayor parte de los indicadores presentaban, en la Evaluación Intermedia del P. O. I., una eficacia en torno o por encima del $30 \%$, lo que en una primera aproximación cabe valorar como un avance positivo de la ejecución, en línea con los propios resultados financieros.

El Cuadro $\mathrm{n}^{\circ} 2$ sintetiza la información financiera relativa a los ámbitos de intervención definidos, y permite conocer, partiendo de la estrategia de desarrollo prevista para el periodo 2000-2002, de qué manera la ejecución alcanzada se ha ajustado a las previsiones iniciales y, en consecuencia, en qué ámbitos cabía esperar una mayor incidencia en los primeros años de aplicación.

\section{Cuadro $\mathrm{n}^{\circ} 1$}

INVERSIÓN PREVISTA, PAGOS REALIZADOS Y EFICACIA FINANCIERA DE LAS MEDIDAS DE DESARROLLO RURAL DEL P. O. I. DE MURCIA

\begin{tabular}{|c|c|c|c|c|c|}
\hline Medidas & $\begin{array}{l}\text { Inversión prevista } \\
\text { 2000-2006 (euros) }\end{array}$ & \begin{tabular}{|c|} 
Peso financiero s/ \\
Eie correspondiente \\
$(9)$
\end{tabular} & $\begin{array}{c}\text { Peso financiero 3/ } \\
\text { total medidas } \\
\text { desarrollo rural (\%) }\end{array}$ & $\begin{array}{l}\text { Pagos realizados } \\
\text { 2000-2002 (euros) }\end{array}$ & \begin{tabular}{|c|} 
Eficacia s/ \\
$2000-2006$ \\
$(\%)$
\end{tabular} \\
\hline Medida 1.2 & 77.841 .332 & 39,03 & 52,42 & 28.046 .727 & 36,03 \\
\hline Medida 3.9 & 18.372 .308 & 2,92 & 12,37 & 8.196 .230 & 44,61 \\
\hline Medida 3.10 & 33.490 .767 & 5,33 & 22,56 & 5.024 .388 & 15,00 \\
\hline Medida 7.2 & 15.000 .001 & 79,87 & 10,10 & 5.603 .183 & 37,35 \\
\hline Medida 7.8 & 3.780 .000 & 20,13 & 2,55 & 970.724 & 25,68 \\
\hline \begin{tabular}{|l|} 
Total medidas \\
desarrollo rural
\end{tabular} & 148.484 .408 & $8,7^{*}$ & 100,00 & 47.841 .252 & 32,22 \\
\hline Total Eje 7 & 18.780 .001 & - & 12,65 & 6.573 .907 & 35,00 \\
\hline
\end{tabular}

* Peso financiero de las medidas de desarrollo rural sobre la inversión total del P. O. I.

Fuente: Evaluación Intermedia del POI de la CARM 


\section{Cuadro $\mathrm{n}^{\circ} 2$ \\ SÍNTESIS DE LOS PRINCIPALES INDICADORES FÍSICOS Y CÁLCULO DE LA EFICACIA DE LAS MEDIDAS DE DESARROLLO RURAL DEL POI DE LA REGIÓN DE MURCIA}

\begin{tabular}{|c|c|c|c|c|c|}
\hline Mida & Indicador & Ud & $\begin{array}{c}\text { Programado } \\
00-06\end{array}$ & $\begin{array}{c}\text { Realizado } \\
00-02\end{array}$ & $\begin{array}{l}\text { Eficacia } \\
\text { s/ 00-06 }\end{array}$ \\
\hline 1.2 & Empresas beneficiarias (Pymes) & $\mathrm{N}^{\circ}$ & 395 & 122 & 30,89 \\
\hline & Empresas beneficiarias & $\mathrm{N}^{*}$ & 415 & 129 & 31,08 \\
\hline & Inversión privada inducida & Euro5 & 182.000 .000 & 81.360 .565 & 44,70 \\
\hline & Inversiones verdes / inversiones emprendidas & $\%$ & 3 & 4.86 & 162,00 \\
\hline & Empleos creados & $\mathrm{N}^{\circ}$ & 500 & 687 & 137,40 \\
\hline & Empleos mantenido5 & $\mathrm{N}^{2}$ & 15.000 & 3.295 & 21,97 \\
\hline 3.9 & Infraestructuras forestales mejoradas & $\mathrm{N}^{*}$ & 30 & 30 & 100,00 \\
\hline & Superficie forestal afectada & Has & 70.000 & 31.489 & 44,98 \\
\hline & Unidades materiales públicas & $\mathrm{N}^{0}$ & 152 & 63 & 41,45 \\
\hline & Vias forestales mejoradas & $\mathrm{Km}$ & 375 & 146 & 38,93 \\
\hline & $\begin{array}{l}\text { Superficie beneficiada por acciones de protección y } \\
\text { restauración de recursos naturales, agua, suelo y } \\
\text { cubierta vegetal }\end{array}$ & Has & 4.961 & 1.833 & 36,95 \\
\hline & Diques (forestales) & $\mathrm{N}^{0}$ & 200 & 2 & 1.00 \\
\hline 3.10 & Areas recreativas & $\mathrm{N}^{\circ}$ & 12 & 8 & 66,67 \\
\hline & Campañas & $\mathrm{N}^{*}$ & 26 & 11 & 42,31 \\
\hline & Espacios protegido5 & $\mathrm{N}^{2}$ & 19 & 13 & 68,42 \\
\hline & Especies protegida5 & $\mathrm{N}^{\circ}$ & 25 & 17 & 68,00 \\
\hline & Estudios realizado5 & $\mathrm{N}^{\circ}$ & 25 & 37 & 148,00 \\
\hline & Superficie considerada & Has & 80.000 & 54.083 & 67,60 \\
\hline & Publicaciones & $\mathrm{N}^{\mathrm{T}}$ & 40 & 8 & 20,00 \\
\hline 7.2 & Caminos rurales construidos o mejorado5 & $\mathrm{Km}$ & 596 & 462 & 77,49 \\
\hline & Superficie afectada & Has & 8.000 & 3.957 & 49,46 \\
\hline & Empleos creados en fase de construcción & $\mathrm{N}^{\mathrm{v}}$ & 360 & 248 & 68,81 \\
\hline 7.8 & $\begin{array}{l}\text { Proyectos de comercialización de productos agricolas } \\
\text { de calidad }\end{array}$ & $\mathrm{N}^{*}$ & 150 & 50 & 33,33 \\
\hline & $\begin{array}{l}\text { Grupos creados de comercialización de productos } \\
\text { agricolas de calidad }\end{array}$ & $\mathrm{N}^{\circ}$ & 2 & 3 & 150,00 \\
\hline & Productos de calidad comercializados & $\mathrm{N}^{*}$ & 15 & 23 & 153,33 \\
\hline & Empleos creado5 & $\mathrm{N}^{0}$ & 10 & 9 & 90 \\
\hline
\end{tabular}

Fuente: Fondos 2000.

Del conjunto de actuaciones realizadas se ponen de relieve las relativas al apoyo a las industrias agroalimentarias, esta decisión se sustenta en que se consideran trascendentes para la estrategia de desarrollo rural programada en el P. O. I., y en la recomendación que se hace, desde la Guía metodológica para la evaluación de las intervenciones de FEOGA, en los programas del Objetivo 1.

Groso modo cabe resaltar que al sector agroalimentario murciano se le reconoce, como al turismo, su capacidad para dinamizar el desarrollo de la Región de Murcia. Su estructuración se basa fundamentalmente en industrias dedicadas a la manipulación de frutas y hortalizas, actividad que desarrollan el 47,1\% de las industrias agroalimentarias de la Región. En segundo y tercer lugar aparece la industria cárnica y las bodegas, sumando entre estos tres sectores el $77,0 \%$ del total. 
Cuadro $\mathrm{n}^{\circ} 3$

PROGRAMA OPERATIVO DE MURCIA. DISTRIBUCIÓN DE LA FINANCIACIÓN POR FUENTES Y EJES DE INTERVENCIÓN 2000-2006 (EN EUROS)

\begin{tabular}{|c|c|c|c|c|c|c|c|c|c|}
\hline \multirow{3}{*}{$\begin{array}{c}\text { Eje } \\
\text { prioritario } \\
\text { de } \\
\text { intervendion }\end{array}$} & \multirow{3}{*}{$\begin{array}{c}\text { Coste } \\
\text { total } \\
\text { elegible }\end{array}$} & \multirow{3}{*}{$\begin{array}{l}\text { Total } \\
\text { pábico } \\
\text { elegible }\end{array}$} & \multicolumn{7}{|c|}{ Participadín páblica } \\
\hline & & & \multicolumn{4}{|c|}{ Comunitaria } & \multicolumn{3}{|c|}{ Nacional } \\
\hline & & & Total & FEDER & FSE & FEOGA & Total & A. Central & A. Regional \\
\hline Eje 1 & 199.462 .606 & 199.462 .606 & 140.817 .925 & 60.480 .000 & 22034.926 & 58380.999 & 58644.681 & 14.066 .000 & 44.578 .681 \\
\hline Eje? & 53.047 .45 & 53.047 .455 & 37.133 .218 & 32.681 .973 & 4.4512245 & & 15914.237 & 460.42 & 15.48 .980 \\
\hline Eje 3 & Q26.161.087 & 626.161027 & 428.463 .746 & 394.752 .745 & & 33.711 .001 & 199.687 .281 & 170.573 .358 & 27.159963 \\
\hline Ege $4 \mathrm{~A}$ & 118.764 .658 & 118.764 .658 & 76.009 .658 & 60.489 .972 & 15.549 .686 & & 42.755 .000 & & 42.7555000 \\
\hline Ege4 B & 51.171 .140 & 51.172 .140 & 33.261 .241 & & 33.261 .241 & & 17.909 .899 & & 17.998999 \\
\hline Ege4C & 12.124 .347 & 12.124.347 & 8.487 .043 & & 8.487 .043 & & 3.637 .304 & & 3.637 .304 \\
\hline Ege40 & 21.633887 & 21.63 .887 & 15.143 .722 & & 15.143 .722 & & 6490.165 & & 6.400 .165 \\
\hline Eje4E & 10.226 .819 & 10.266819 & 7.67 .113 & & 7.60 .113 & & 2.566 .706 & & 2.556 .706 \\
\hline Eje 5 & 167.599 .370 & 167.509 .370 & 114.202275 & 116.200275 & & & 53.229 .095 & 34.366 .565 & 18.922 .530 \\
\hline Eje6 & 425.663 .375 & 425.668 .375 & 257.928 .007 & 257.98 .07 & & & 167.681 .368 & 134.75 .241 & 32.06 .127 \\
\hline Ege 7 & $18.700,011$ & 18.700001 & 13.146000 & & & 13.146.000 & 5634001 & & 5.634 .001 \\
\hline Aistencia Térnica & 6.5320067 & 6.52 .067 & 4.999 .052 & 3.141008 & 1.200 .024 & 566.000 & 1633.015 & 185.334 & 1.447 .681 \\
\hline Total & 1.711.076.752 & 1.711.076.752 & 1.137.294.000 & 923.000 .000 & 107.8000 .000 & 105.794 .000 & 573.782 .752 & 354335.927 & 219.446 .825 \\
\hline
\end{tabular}

Fuente: Dirección General de Fondos Comunitarios y Financiación Territorial. $\mathbf{M}^{\mathrm{o}}$ de Hacienda.

El Programa Operativo 2000-2006, ha sido elaborado según el principio de cooperación, es decir, en estrecha colaboración entre los servicios de la Comisión Europea y las autoridades competentes a escala nacional y regional. Los recursos a aplicar proceden del FEDER, FSE y FEOGA-O, aunque es el FEDER el que proporciona más de las cuatro quintas partes del conjunto de la ayuda (Cuadro $\mathrm{n}^{\circ} 3$ ). La mayor parte de la cofinanciación, $61,75 \%$, se instrumenta a través de la Administración General del Estado y un 38,25\% por la Administración Regional.

Existen informes anuales del P. O. I. de la Región de Murcia. En concreto disponemos del Informe de Ejecución Anual de 2004 que nos permite observar, entre otros aspectos, las siguientes cuestiones. En primer lugar, la situación en la que se encuentra la aplicación de los ejes prioritarios y medidas; en segundo lugar, su ejecución financiera y, en tercer lugar, las disposiciones adoptadas para garantizar la calidad de las intervenciones. De igual modo resaltamos el apartado dedicado a las medidas adoptadas para garantizar la compatibilidad con las políticas comunitarias.

Si nos referimos exclusivamente a la cuestión que nos ocupa, el eje 7 «agricultura y desarrollo rural», cabe señalar que el porcentaje de ejecución para el año 2004, alcanzaba un 76,08\% y la ejecución de los años 2000-2004 un 92,79\%. Dentro de este Eje, la Comunidad Autónoma de Murcia, lleva a cabo las medidas 7.2 y 7.8, que presentamos en sus respectivos cuadros $\mathrm{n}^{\circ} 4 \mathrm{y} \mathrm{n}^{\circ} 5$, cofinanciadas ambas por el FEOGA-O. Durante el ejercicio 2004 se realizaron actuaciones por importe de 2.418.397,84 euros, que suponen ese grado de ejecución del 76,08 \% de la anualidad prevista. 
Además de los procedimientos que se ajustan estrictamente al eje «agricultura y desarrollo rural», consideramos que existen acciones en otros ejes que también favorecen claramente a los espacios rurales de la Región. En este sentido, destacamos medidas del eje 3 «medio ambiente, entorno natural y recursos hídricos» y, de forma más nítida, la medida 9 «silvicultura», en la que se han llevado a cabo Proyectos de tratamientos silvícolas; Proyectos de reforestaciones para restauración: de laderas y terrenos agrícolas abandonados, de una escombrera en monte público y de riberas en la Cuenca del Noroeste de la Región a fin de proteger y mejorar el suelo y disminuir la erosión hídrica; Proyectos de mejora y acondicionamiento de caminos forestales para su adecuación como vía de penetración en caso de incendio, para mejorar las condiciones de tránsito de los montes y para facilitar la gestión forestal de los mismos; Proyectos de adecuación de infraestructuras forestales en montes de utilidad pública, etc. Además, para facilitar la ordenación forestal así como la planificación de las obras de selvicultura preventiva contra los incendios forestales, se han elaborado numerosos estudios y trabajos técnicos.

Esta Medida tiene como finalidad principal la conservación de áreas protegidas, la conservación y recuperación de la flora y fauna silvestre, y la adecuación del uso público en espacios naturales. Su ejecución financiera está compartida, según se establece en el Programa Operativo Integrado, al 50\% con la Dirección General para la Biodiversidad del Ministerio de Medio Ambiente, que cofinancia actuaciones en el concepto presupuestario «Programas de Red Natura 2000, Patrimonio natural y paisaje, difusión y formación en Conservación de la Biodiversidad».

\subsection{LEADER+ en la Región de Murcia}

Con este Programa Operativo, coincide en el tiempo la Iniciativa LEADER $+^{2}$ cuyas directrices quedaron establecidas en la Comunicación de la Comisión a los Estados miembros 2000/ C139/05 (DOCE $\mathrm{n}^{\circ} \mathrm{C} 139$, de 18 de mayo de 2000). Una importante novedad, respecto a las fases anteriores, es su amplia cobertura territorial. Sus acciones se extienden por los siguientes espacios de la Región:

- Territorio de la Comarca del Altiplano: situada en el Nordeste de la Región y formada por los municipios de Abanilla, Fortuna, Jumilla y Yecla.

- Territorio de la Comarca del Noroeste, situada en el Noroeste de la Región y compuesta por los municipios de Caravaca de la Cruz, Moratalla, Cehegín y Bullas.

- Territorio de la Comarca de la Vega Alta del Río Segura, ubicada en la Vega del Río Segura y formada por los municipios de Calasparra, Cieza, Abarán, Blanca, Archena, Ojós, Ricote, Ulea, Alguazas, Ceutí, Lorquí y Villanueva del Río Segura.

- Territorio de la Comarca del Río Mula, situada junto al río del mismo nombre y comprende los municipios de Mula, Pliego, Albudeite y Campos del Río.

- Territorio de la Comarca del Valle de Guadalentín, de quien recibe el nombre, y que está compuesto por los municipios de Aledo y Puerto Lumbreras, así como de parte de los términos municipales de Lorca (concretamente en su sector septentrional comprendido por las diputaciones y pedanías siguientes: Avilés, Béjar, Coy, Culebrina,

2 CONSEJERÍA DE AGRICULTURA, AGUA Y MEDIO AMBIENTE: Orden de 2 de octubre de 2001 por la que se establecen las normas para la presentación y planes de desarrollo rural para su aplicación en territorios pertenecientes a la Comunidad Autónoma de la Región de Murcia en el marco de la Iniciativa Comunitaria LEADER +. 


\section{Cuadro $\mathrm{n}^{\circ} 4$ \\ FICHA TÉCNICA DE MEDIDA}

\section{Programa Operativo: Murcia 2000-2006 Objetivo: 1 \\ Eje: 7 AGRICULTURA Y DESARROLLO RURAL \\ Medida: 2 Desarrollo y mejora de las infraestructuras de apoyo}

Por medidas, en el año 2004, las inversiones de la Dirección General de Regadíos y Desarrollo Rural en esta medida ascienden a un total de 1.790.209,08 euros, alcanzando un 67,33\% de lo programado para el ejercicio. La ejecución acumulada 2000-2004 es de 11.196.190,39 euros, representando un $98,74 \%$ sobre el total previsto para el período. La medida está encaminada a la mejora y mantenimiento de las comunicaciones y vías de acceso a las explotaciones agrarias, así como a los caseríos y viviendas rurales diseminadas. Con ello se pretende dar una mayor permeabilidad a las zonas rurales, contribuyendo significativamente al desarrollo endógeno de las mismas.

Destacan las siguientes actuaciones durante 2004:

- Acondicionamiento de caminos rurales de servicio en Fortuna.

- Acondicionamiento de caminos en el T.M. de Blanca

- Caminos rurales en las Vegas del Segura $3^{\text {a }}$ Fase.

- Acondicionamiento de caminos en la Zona Regable V y VI de las Vegas de Lorca y Valle del Guadalentín $2^{\text {a }}$ Fase T.M. de Totana.

- Acondicionamiento de caminos en las Zonas Regables V y VI de las Vegas de Lorca y Valle del Guadalentín $3^{\text {a }}$ Fase T.M. de Totana.

Proyectos con ejecución finalizada y pagos pendientes

- Acondicionamiento de caminos en la Zona Regable del T.M. de Torre Pacheco. Ha finalizado y se han recibido las obras de este proyecto.

- Acondicionamiento de caminos rurales en Mula. Ha finalizado y se han recibido las obras de este proyecto.

- Acondicionamiento de caminos en las Torres de Cotillas. Este proyecto se ha iniciado y finalizado la ejecución del mismo en 2004.

- Acondicionamiento de caminos rurales del Plan Integral del Noroeste - Río Mula $1^{\text {a }}$ Fase TT.MM. de Albudeite y Campos del Río.

- Acondicionamiento de caminos Plan Integral Noroeste $2^{\text {a }}$ Fase.

- Acondicionamiento de caminos en Águilas, Lorca (Sur) y Mazarrón.

- Acondicionamiento de caminos en Lorca.

Proyectos en ejecución

- Acondicionamiento de caminos rurales de servicio en el T.M. de Cieza.

- Acondicionamiento de caminos en Lorca $2^{\mathrm{a}}$ Fase.

- Acondicionamiento de caminos rurales del Plan de Desarrollo Integral del Noroeste

- Río Mula 1ª Fase en los TT.MM. de Bullas y Cehegín.

Fuente: Informe de Ejecución Anual de 2004 P. O. Región de Murcia 2000-2006 


\section{Cuadro $\mathrm{n}^{\circ} 5$ \\ FICHA TÉCNICA DE MEDIDA}

\section{Programa Operativo: Murcia 2000-2006 Objetivo: 1} Eje: 7 AGRICULTURA Y DESARROLLO RURAL

Medida: 8 Prestación de servicios a las explotaciones agrarias, comercialización de productos agrarios de calidad e ingeniería financiera.

Potenciación de Denominaciones de Origen específicas y genéricas.

Apoyo a las Organizaciones Interprofesionales Agroalimentarias para la comercialización de productos de calidad.

Apoyo a las Agrupaciones de empresas, incluso empresas, que inicien actuaciones destinadas a la implantación de sistemas de aseguramiento de la calidad y al desarrollo de denominaciones de calidad.

Durante el ejercicio 2004 se realizan pagos por importe de $628.188,76 €$, lo que supone un grado de ejecución del 120,83\%. Los pagos acumulados en el período 2000-2004 ascienden a la cantidad de $1.837 .743,04 €$, que comparado con el gasto elegible para el mismo período supone una ejecución del $67,88 \%$, por lo que se puede considerar que el nivel de ejecución es bueno, teniendo en cuenta que faltan 85 expedientes con cargo al Presupuesto de 2004 cuyo pago efectivo se realizará en el 2005.

Se tramitan 57 proyectos cuyo pago efectivo se efectuava en el periodo comprendido entre el 01-01-2004 y 31-12-2004, cuya distribución es la siguiente: 9 Consejos reguladores; 4 Asociaciones y/o agrupaciones y 44 empresas.

En esta medida, a lo largo del ejercicio 2004, se efectúan pagos por importe de $628.188,76 €$, lo que representa un grado de ejecución del 120,83\%. En el período 2000-2004 los pagos acumulados ascienden a 1.837.743,04 €, siendo esta cantidad un $67,88 \%$ del total de gasto elegible.

Fuente: Informe de Ejecución Anual de 2004 P. O. Región de Murcia 2000-2006.

Doña Inés, Fontanares, Umbrías, Jarales, Nogalte, Ontillo, La Paca, Torrealvilla, La Tova, Zarcilla de Ramos, Zarzadilla de Totana y Zarzalico). Del municipio de Alhama de Murcia (zona de Sierra Espuña integrada por Sierra Espuña, El Berro y Gebas) y en el caso de Totana (también en la zona de Sierra Espuña compuesta por Sierra Espuña, Morti y Las Viñas).

- Territorio de la Comarca del Campo de Cartagena, integrada por el término municipal de Fuente Álamo, el sector noroccidental del municipio de Cartagena (comprendido por La Aljorra, Perín y Los Huertos y el sector suroriental del término de Murcia (integrado por Baños y Mendigo, Carrascoy, Corvera, Lobosillo, Valladolises y Lo Jurado), correspondiendo a una planicie situada en zona no regable. 
La aplicación de la Iniciativa Leader+ se conforma y concreta a partir de cuatro planes comarcales, cada uno con su propio G. A. L. ${ }^{3}$, que se ajustarán a lo dispuesto en la Comunicación de la Comisión 2000/ C 139/2005, por la que se fijan las orientaciones sobre la Iniciativa, y en el Programa Regional aprobado por Decisión de la Comisión $n^{\circ}$ C(2001) 2183, de 23 de agosto de 2001. Dichos planes cuentan con sus correspondientes asignaciones financieras para las distintas medidas subvencionables. En la evaluación que se ha realizado, se constata la diversidad de enfoques en la asignación de recursos a cada medida, lo que pone de manifiesto la diferente perspectiva que cada G. A. L. adjudica a la Iniciativa. Así, mientras que el Grupo Vega del Segura realiza una distribución más lineal entre las distintas medidas, otros Grupos han priorizado la asignación a la medida de turismo ante la fuerte demanda de proyectos relacionados con el turismo rural. Esta situación ha llevado a recomendar mayor cuidado en mantener la perspectiva global de lo que se quiere conseguir con el programa y no actuar bajo presión de la demanda. Los resultados observados son los siguientes:

- Concentración de proyectos en algunas medidas, pero sobre todo en turismo rural y muy escasa representación de otras medidas, como es el caso de patrimonio natural o valorización de productos agrarios.

- Número sensiblemente alto de proyectos presentados. Con informe de elegibilidad positivo hay 140 proyectos y con informe pendiente otros 121 proyectos.

- Concentración de proyectos en dos Grupos: Integral y Vega del Segura. Bajo dinamismo en Nordeste y escasa representación en el Grupo Campoder. Con ello la evolución de la Iniciativa se encuentra sesgada y concentrada sobre todo en dos territorios que son los que están definiendo los resultados regionales.

- Elevados volúmenes en cuanto al valor de los presupuestos presentados. Sólo con los contenidos en los informes de elegibilidad positivos se cubre el $100 \%$ de lo previsto para todo el periodo de programación de la Iniciativa, en términos de costes totales. Con todo, se observan grados de ejecución muy bajos en algunos Grupos, sobre proyectos contratados y además son aquellos Grupos en los que menos proyectos tienen pendientes de contratación. En ellos se debe hacer un esfuerzo para la dinamización de la Iniciativa, en tanto que en los otros dos Grupos el esfuerzo se ha de hacer en la priorización y selección de proyectos, al menos en la fase actual de información y contratación. En definitiva, se recomienda la realización de un análisis detallado del estado de la Iniciativa, en términos

3 Resolución de 26 de Noviembre de 2002, por la que se publica el Convenio entre el Organismo Intermediario, constituido por el Ministerio de Agricultura, Pesca y Alimentación y la Consejería de Agricultur,a Agua y Medio Ambiente, y el Grupo de Acción Local «Asociación para el desarrollo rural integrado de la Vega del Segura», para la aplicación de la Iniciativa Comunitaria Leader Plus.

Resolución de 26 de Noviembre de 2002, por la que se publica el Convenio entre el Organismo Intermediario, constituido por el Ministerio de Agricultura, Pesca y Alimentación y la Consejería de Agricultur,a Agua y Medio Ambiente, y el Grupo de Acción Local «Asociación para el Desarrollo Integral del Campo de Cartagena de la Región de Murcia», para la aplicación de la Iniciativa Comunitaria Leader Plus.

Resolución de 26 de Noviembre de 2002, por la que se publica el Convenio entre el Organismo Intermediario, constituido por el Ministerio de Agricultura, Pesca y Alimentación y la Consejería de Agricultura Agua y Medio Ambiente, y el Grupo de Acción Local «Asociación para el desarrollo comarcal del Nordeste de la Región de Murcia», para la aplicación de la Iniciativa Comunitaria Leader Plus.

Resolución de 26 de Noviembre de 2002, por la que se publica el Convenio entre el Organismo Intermediario, constituido por el Ministerio de Agricultura, Pesca y Alimentación y la Consejería de Agricultura Agua y Medio Ambiente, y el Grupo de Acción Local «Integral, sociedad para el desarrollo rural», para la aplicación de la Iniciativa Comunitaria Leader Plus. 
financieros, con objeto de conocer con exactitud su situación y realizar así las proyecciones oportunas.

No obstante lo anterior, el evaluador considera que, en líneas generales, la evolución de los territorios ha sido positiva, centrada en un buen dinamismo económico general, tanto territorial como regional, así como acompañada con un crecimiento poblacional singularmente intenso, en algunas zonas que obedece al fenómeno migratorio que afecta a la Región de Murcia.

\section{A modo de conclusión. Perspectivas de futuro del desarrollo rural en la Región de Murcia}

La Conferencia Europea sobre el desarrollo rural ${ }^{4}$ considera que el futuro de la política de desarrollo rural debe guiarse por los siguientes principios:

1. Un campo vivo no sólo redunda en beneficio de la sociedad rural sino también en el de la sociedad en su conjunto. La inversión en la economía rural y en las comunidades rurales en sentido amplio, es crucial para aumentar el atractivo de las zonas rurales, promover el crecimiento sostenible y generar nuevas oportunidades de empleo, especialmente entre las mujeres y los jóvenes.

2. La preservación de la diversidad del campo europeo, y el estímulo de los servicios que puede prestar la agricultura multifuncional, revisten cada vez mayor importancia. Gestionar el entorno agrario y los bosques servirá para preservar y mejorar el paisaje natural y el variado patrimonio cultural de Europa, especialmente en las zonas rurales más alejadas dotadas de parajes de gran valor natural.

3. La competitividad del sector agrario debe ser un objetivo fundamental para el que deberá tenerse presente la diversidad del potencial agrario de las distintas zonas rurales. Este aspecto reviste especial importancia en los nuevos Estados miembros dada la significativa reestructuración agraria que es de prever en ellos. En todos los Estados miembros el crecimiento económico sostenible del sector agrario deberá depender cada vez más de la diversificación, la innovación y los productos de valor añadido que demandan los consumidores.

4. La política de desarrollo rural debe aplicarse en todas las zonas rurales de la Unión Europea ampliada para que los agricultores y otros actores del ámbito rural puedan hacer frente a los desafíos de la reestructuración del sector agrícola en curso, de los efectos de la reforma de la PAC y de la evolución de las pautas del comercio en el sector ${ }^{5}$.

En consonancia con estos principios, para el período de programación 2007-2013, la política de desarrollo rural, en el marco de los objetivos fijados por el Reglamento (CE) $\mathrm{n}^{\circ} 1698 / 2005$, se centra en tres ámbitos fundamentales: la economía agroalimentaria, el medio ambiente y la economía y la población rurales, en sentido amplio. La nueva generación de estrategias y programas de desarrollo rural se articulará en torno a cuatro ejes: eje 1, aumento de la competitividad del sector agrícola y forestal; eje 2, mejora del medio

4 Reunida en Salzburgo del 12 al 14 de noviembre de 2003 para evaluar la aplicación de la política de desarrollo rural de la Unión Europea a partir de la Agenda 2000 y prever las necesidades futuras.

5 http://europa.eu.int/comm/agriculture/events/salzburg/index 
ambiente y del entorno rural; eje 3, calidad de vida en las zonas rurales y diversificación de la economía rural; eje 4, LEADER.

Resulta obligado, por lo tanto, que la Región de Murcia se adapte a estas exigencias en su programación para el citado periodo. En este sentido, el gran reto para el futuro es el de la implementación de un Programa Estratégico que, en concordancia con el próximo Programa Operativo de la Unión Europea, se sitúa en el marco 2007-2013. El contenido de dicho Programa Estratégico se elabora a partir de los resultados obtenidos con la Iniciativa «Horizonte 2010» por la que se invitó a los murcianos a que se impliquen activamente en este proceso, aportando sus ideas y opiniones, tanto sobre los temas generales que fueron proponiendo, como sobre los avances del Plan que vayan encontrando y sean sometidos a su consideración.

La Iniciativa ha reunido nueve «grupos de contraste» - uno de cada comarca - formados por dos representantes de cada municipio. También se ha contado en esta iniciativa con las aportaciones de expertos sobre los principales temas que influyen en el desarrollo de esta Comunidad Autónoma. El «Horizonte 2010» ha celebrado treinta debates dentro de la formula de participación «Mesas de Trabajo». Estas mesas, divididas por su temática en Transversales, Verticales y Complementarias, han trabajado en varias sesiones sobre aspectos tales como Ordenación del Territorio, Infraestructuras y Transportes, Empleo y Formación, I+d+i y Sociedad del Conocimiento, Desarrollo Rural, Recursos Hídricos, Igualdad de Oportunidades, Inmigración etc. En definitiva, el gran objetivo es conseguir que la Región de Murcia sea una región líder europea en aquellos ámbitos que le permitan aumentar su competitividad y atractivo y su alcance se concreta en los siguientes objetivos estratégicos: Crecimiento y empleo en los complejos productivos prioritarios de la Región; aumento de la competitividad y productividad; sostenibilidad territorial y ambiental, y cohesión social.

Como puede observarse, el desarrollo rural sigue siendo una cuestión relevante de cara al futuro de esta Comunidad Autónoma. De ahí se desprende que los esfuerzos realizados hasta el presente no han conseguido erradicar el conjunto de causas que generan las desigualdades entre los diferentes espacios de la Región de Murcia. Por esa razón, debemos ser más exigentes, y excluir de la gestión de los fondos procedentes de las distintas administraciones, con quienes han asumido voluntariamente la responsabilidad de optimizar unos recursos que significan el esfuerzo de todos los miembros de la Unión Europea. No obstante, esos fallos no deben empañar las acciones positivas que se han acometido en los últimos años, desde las distintas administraciones implicadas, porque han servido para paliar algunos de los problemas detectados a principios de los noventa y para dinamizar territorios que se encontraban en situación de declive socioeconómico.

\section{Bibliografía}

ANDRÉS SARASA, J. L. (2004): «El proceso desrulalizador de la Región de Murcia: sus consecuencias». Nimbus $\mathrm{n}^{\circ} 13-14$, pp. 33-70.

B. O. E. núm. 19 de 22 enero de 2004. ORDEN APA/64/2004, de 15 de enero, por la que se establecen las normas para la presentación y selección de proyectos de cooperación en el marco de la iniciativa comunitaria LEADER PLUS.

COM (2005) 304 final: Propuesta de DECISIÓN DEL CONSEJO sobre las directrices estratégicas comunitarias de desarrollo rural (Período de programación de 2007-2013).

COMISIÓN EUROPEENNE (1997): Evolutions du monde rural: PAC 2000, Document de Travail. Direction Générale pour l'Agriculture (DG VI). Juillet 1997.

COMISIÓN DE LAS COMUNIDADES EUROPEAS: El futuro de las iniciativas comunitarias al amparo de los Fondos Estructurales. Bruselas, 16.03.94. 
COMISIÓN DE LAS COMUNIDADES EUROPEAS: Decisión núm. C (2001) 2183 de 23 de agosto de 2001.

CONSEJERÍA DE ECONOMÍA INDUSTRIA E INNOVACIÓN. Plan Integral de Desarrollo de las comarcas del Noroeste y Río Mula 2004 - 2009.

CONSEJO ECONÓMICO Y SOCIAL DE LA REGIÓN DE MURCIA, (2000): Informe sobre la reforma de la PAC y el sector agrario de la Región de Murcia. $\mathrm{N}^{\circ} 1$.

DG AGRI, October 2005: Community Strategic Guidelines 2007-2013 Rural Development.

DO L 161 de 26.6.1999

DOCE N ${ }^{\circ}$ C 94 180/12 de 1 de julio de 1994

DOCE L 161, 26.6.99: Reglamento (CE) n ${ }^{\circ}$ 1260/1999 del Consejo de 21 de junio de 1999 por el que se establecen disposiciones generales sobre los Fondos Estructurales.

DOCE C 51 de 23.2.2000: Dictamen del Comité Económico y social sobre el «Proyecto de Comunicación de la Comisión a los Estados miembros por la que se fijan orientaciones sobre la Iniciativa Comunitaria de desarrollo rural (Leader+); C (2000) 946 final. Comunicación de la Comisión a los Estados miembros por la que se fijan orientaciones sobre la iniciativa de desarrollo rural (LEADER+)

DOCE C 234/32, 22.9.2005: Dictamen del Comité Económico y Social Europeo sobre la «Propuesta de Reglamento del Consejo relativo a la ayuda al desarrollo rural a través del Fondo Europeo Agrícola de Desarrollo Rural (FEADER)»

DOCE L 55/20, 25.2.2006: DECISIÓN DEL CONSEJO de 20 de febrero de 2006 sobre las directrices estratégicas comunitarias de desarrollo rural (período de programación 2007-2013) (2006/144/CE)

ESPEJO MARÍN, C. y MENDOZA MARTÍNEZ, J. L. (2003): «Trabajadoras asalariadas en el campo murciano». Papeles de Geografía $\mathrm{n}^{\circ}$ 37. pp. 55-63.

MILLAN ESCRICHE, M. (2000): «Iniciativas de las Comunidades Europeas para el desarrollo local». Nimbus $\mathrm{n}^{\circ}$ 5-6 Enero-Diciembre. Pp.43-53.

MILLÁN ESCRICHE, M. (2002): «La diversificación en el medio rural como factor de desarrollo. Papeles de Geografía no 36. Pp. 223-238.

MILLÁN ESCRICHE, M. (2004): «La Geografía de la Percepción: una metodología de análisis para el desarrollo rural». Papeles de Geografía. $N^{o}$ 40. Pp. 133-149.

MILLÁN ESCRICHE, M. (2004): La Iniciativa Comunitaria LEADER en el desarrollo local del Noroeste murciano. Servicio de Publicaciones de la Universidad de Murcia.

MILLÁN ESCRICHE, M. (2005): «Reflexiones para el estudio de modelos de desarrollo del turismo de interior. El caso de la Región de Murcia». I Congreso de Turismo de Interior y Montaña. www.lleidatur.com.

MILLÁN ESCRICHE, M. (2005): «Los retos de los actores del desarrollo local en el tránsito de la tradición a la innovación»V Jornadas de Desarrollo Local. Alicante

MINISTERIO DE ECONOMÍA Y HACIENDA (Septiembre 2005): P. O. I. DE MURCIA 2000-2006 Objetivo $n^{\circ} 1$ FEDER, FEOGA-O, FSE INFORME DE EJECUCIÓN Anualidad 2004. 